\title{
Nota sobre la interpretación de diagnósticos de PAAF de mama
}

\section{Sr. Director:}

Como patólogos con experiencia en distintos hospitales de la geografía nacional creemos que es éste un foro adecuado para realizar las siguientes especificaciones a modo de respuesta a las continuas peticiones de nuestros compañeros clínicos, y en especial oncólogos, que muestran cierto desconcierto ante los distintos términos usados como diagnóstico para las muestras obtenidas mediante PAAF, en especial aquellas procedentes de lesiones mamarias. $\mathrm{Si}$ bien cada patólogo tiene su peculiar criterio para informar este tipo de muestras, está bien extendido el uso de una gradación terminológica que no siempre facilita la comprensión de quien posteriormente la interpreta. Estamos todos de acuerdo en lo que un diagnóstico de "benignidad" o de "ausencia de malignidad" representa, añadiendo por supuesto la consideración de "material no adecuado, valorable o representativo" para aquellas muestras que necesitarán de una repetición para ser correctamente valoradas pues no se trata de un diagnóstico sinónimo de benignidad. El gran problema surge cuando se observan signos que pueden ser interpretados como neoplásicos. Se utiliza el término de "compatible" cuando los hallazgos observados se corresponden con los del juicio clínico previo siempre que no existan datos que nos hagan emitir un diagnóstico de certeza. El término "sospechoso o sugestivo" es utilizado con el fin de transmitir al clínico la necesidad de un estudio más completo (histología generalmente) con el fin de alcanzar un diagnóstico de certeza previo a la decisión terapéutica. Si bien existen falsos positivos, el subsiguiente estudio ayudará a reducirlos a su mínima expresión. Cuando el diagnóstico emitido sea "indicativo de malignidad o neoplasia" podemos asegurar que la muestra cumple los criterios citológicos de malignidad y por tanto este diagnóstico puede ser empleado con las mismas garantías que un diagnóstico de positividad histológico si bien, al igual que en este último, existe un porcentaje de falsos positivos que es muy difícil de reducir aun recurriendo a estudios intraoperatorios. Esta última situación comprendemos que es indeseable por muy pequeño que sea el porcentaje de casos incluidos en este grupo si bien, como defensa de la técnica y del diagnóstico citológico en general, podemos afirmar que estos ejemplos de falsos positivos generalmente se corresponden con lesiones mamarias que si bien no son neoplásicas sí que pueden englobarse en el conjunto de lesiones proliferativas o lesiones de riesgo que por tanto pueden igualmente beneficiarse de una intervención. Creemos fundamental hacer hincapié en estas especificaciones pues son muchos los diagnósticos de este tipo los que se manejan en nuestra práctica diaria. Es conveniente ser precavido y leer siempre las posibles notas que puedan acompañar al informe pues en ellas se encuentra, en la mayoría de los casos, la clave para la correcta interpretación de diagnósticos que en un principio pueden despertar la incertidumbre con respecto a la correspondiente elección terapéutica.

\section{Bibliografía}

1. Geisinger KR, Stanley MW, Raab SS y cols. Modern Cytopathology. Churchill Livingstone, Elsevier Science (USA) 2004.

2. Rodríguez Costa J, De Agustín Vázquez D. Punción Aspiración con Aguja Fina de Órganos Superficiales y Profundos. Díaz de Santos 1997.

\section{F. J. Torres Gómez ${ }^{1}$, M. Díaz Delgado², F. J. Torres Olivera ${ }^{3}$ Servicios de Anatomía Patológica ${ }^{1}$ Hospital Punta de Europa de Algeciras \\ ${ }^{2}$ Hospital de Mérida \\ ${ }^{3}$ Hospital Universitario Virgen Macarena de Sevilla}

Correspondencia: Dr. F. J. Torres Gómez Matahacas, 18 A - 1b E-41003 Sevilla javiertorresgomez@yahoo.es 